Flavia Giammarino and Pauline Barrieu

Department of Statistics, London School of Economics, UK

Email: f.giammarino@lse.ac.uk

\begin{abstract}
We consider the indifference valuation of an uncertain monetary payoff from the perspective of an uncertainty averse decision-maker. We study how the indifference valuation depends on the decision maker's comparative uncertainty attitudes, and we obtain a characterization of increasing, decreasing, and

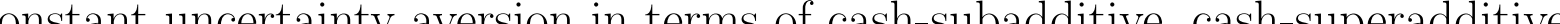
and cash-additive quasiconvex risk measures.
\end{abstract}

\section{Uncertainty Averse Preferences}

An uncertainty averse decision maker evaluates the relative desirability of alternative uncertain monetary payoffs by a functional $U$ on $\mathcal{X}:=$ $B(\Omega, \mathcal{F})$ which is monotone increasing and quasiconcave (see Cerreia Vioglio et al. (2008a))

Assumption 1. A preference functional $U: \mathcal{X} \rightarrow \mathbb{R}$ satisfies the following conditions for all $X, Y \in \mathcal{X}$ and $\lambda \in[0,1]$.

- Increasing monotonicity: If $X>Y$, then $U(X)>U(Y)$.

- Quasiconcavity: $U(\lambda X+(1-\lambda) Y) \geq \min \{U(X), U(Y)\}$.

The properties of increasing monotonicity and quasiconcavity of $U$ on $\mathcal{X}$ can be equivalently characterized in terms of the following representation.

Theorem 1 (Cerreia Vioglio et al. (2008b)). A monotone increasing, quasiconcave, and continuous functional $U: \mathcal{X} \rightarrow \mathbb{R}$ has the following representation

$$
U(X)=\inf _{Q \in \mathcal{M}_{1, f}:=b a_{1}^{+}(\Omega, \mathcal{F})} V\left(\mathbb{E}_{Q}[X], Q\right)
$$

for all $X \in \mathcal{X}$

The representation in Equation (1) implies that an uncertainty averse decision maker evaluates the relative desirability of an uncertain monetary payoff in $\mathcal{X}$ as if, by the function $V$, she appraised its expected value under each probabilistic scenario in $\mathcal{M}_{1, f}$ and as if, by the functional $U$, she summarized her appraisal by considering exclusively the worst scenario in $\mathcal{M}_{1, f}$

\section{Indifference Buyer Price}

The indifference buyer price, considered from an actuarial perspective, is the maximum price that a decision maker with uncertainty averse preferences $U$ and with constant initial wealth $w_{0} \in \mathbb{R}$ would pay to avoid an uncertain monetary payoff in $\mathcal{X}$ (e.g. to receive insurance).

Definition 1. A functional $\pi_{w_{0}}^{U}: \mathcal{X} \rightarrow \mathbb{R}$ is said to be an indifference buyer price if

$$
U\left(w_{0}-\pi_{w_{0}}^{U}(X)\right)=U\left(w_{0}+X\right)
$$

for all $X \in \mathcal{X}$ and $w_{0} \in \mathbb{R}$.

Proposition 1. An indifference buyer price $\pi_{w_{0}}^{U}: \mathcal{X} \rightarrow \mathbb{R}$ satisfies the following properties for all $X, Y \in \mathcal{X}, \lambda \in[0,1]$, and $m \in \mathbb{R}$. - Decreasing monotonicity: If $X>Y$, then $\pi_{w_{0}}^{U}(X)<\pi_{w_{0}}^{U}(Y)$. - Quasiconvexity: $\pi_{w_{0}}^{U}(\lambda X+(1-\lambda) Y) \leq \max \left\{\pi_{w_{0}}^{U}(X), \pi_{w_{0}}^{U}(Y)\right\}$. - Normalization: $\pi_{w_{0}}^{U}(-m)=m$

Proposition 1 implies that $\pi_{w_{0}}^{U}$ is a quasiconvex risk measure. The properties of decreasing monotonicity and quasiconvexity of $\pi_{w_{0}}^{U}$ on $\mathcal{X}$ can be equivalently characterized in terms of the following representation. Proposition 2. An indifference buyer price $\pi_{w_{0}}^{U}: \mathcal{X} \rightarrow \mathbb{R}$ has the following representation

$$
\pi_{w_{0}}^{U}(X)=\sup _{Q \in \mathcal{M}_{1, f}} R_{w_{0}}^{U}\left(\mathbb{E}_{Q}[-X], Q\right)
$$

for all $X \in \mathcal{X}$

The representation in Equation (3) implies that an uncertainty averse decision maker evaluates the maximum price that she would pay to avoid an uncertain monetary payoff in $\mathcal{X}$ as if, by the function $R_{w_{0}}^{U}$, she appraised its expected loss under each probabilistic scenario in $\mathcal{M}_{1, f}$, the appraisal $R_{w_{0}}^{U}$ depending on her uncertainty attitudes $U$ and on her initial wealth $w_{0} \in \mathbb{R}$, and as if, by the functional $\pi_{w_{0}}^{U}$, she summarized her appraisal by considering exclusively the worst scenario in $\mathcal{M}_{1, f}$.

\section{$3 \quad$ Indifference Seller Price}

The indifference seller price, considered from an actuarial perspective, is the minimum price that a decision maker with uncertainty averse preferences $U$ and with constant initial wealth $w_{0} \in \mathbb{R}$ would demand to accept an uncertain monetary payoff in $\mathcal{X}$ (e.g. to provide insurance). Definition 2. A functional $\phi_{w_{0}}^{U}: \mathcal{X} \rightarrow \mathbb{R}$ is said to be an indifference seller price if

$$
U\left(w_{0}+X+\phi_{w_{0}}^{U}(X)\right)=U\left(w_{0}\right)
$$

for all $X \in \mathcal{X}$ and $w_{0} \in \mathbb{R}$.

Proposition 3. An indifference seller price $\phi_{w_{0}}^{U}: \mathcal{X} \rightarrow \mathbb{R}$ satisfies the following properties for all $X, Y \in \mathcal{X}, \lambda \in[0,1]$, and $m \in \mathbb{R}$.

- Decreasing monotonicity: If $X>Y$, then $\phi_{w_{0}}^{U}(X)<\phi_{w_{0}}^{U}(Y)$.

- Convexity: $\phi_{w_{0}}^{U}(\lambda X+(1-\lambda) Y) \leq \lambda \phi_{w_{0}}^{U}(X)+(1-\lambda) \phi_{w_{0}}^{U}(Y)$

- Cash-additivity: $\phi_{w_{0}}^{U}(X+m)=\phi_{w_{0}}^{U}(X)-m$.

- Normalization: $\phi_{w_{0}}^{U}(0)=0$.

Proposition 3 implies that $\phi_{w_{0}}^{U}$ is a cash-additive convex risk measure. The properties of decreasing monotonicity, convexity, and cashadditivity of $\phi_{w_{0}}^{U}$ on $\mathcal{X}$ can be equivalently characterized in terms of the following representation.

Proposition 4. An indifference seller price $\phi_{w_{0}}^{U}: \mathcal{X} \rightarrow \mathbb{R}$ has the following representation

$$
\phi_{w_{0}}^{U}(X)=\sup _{Q \in \mathcal{M}_{1, f}}\left(\mathbb{E}_{Q}[-X]-\alpha_{w_{0}}^{U}(Q)\right)
$$

for all $X \in \mathcal{X}$.

The representation in Equation (5) implies that an uncertainty averse decision maker evaluates the minimum price that she would demand to accept an uncertain monetary payoff in $\mathcal{X}$ as if, by the function $\alpha_{w_{0}}^{U}$, she applied a correction to its expected loss under each probabilistic scenario in $\mathcal{M}_{1, f}$, the correction $\alpha_{w_{0}}^{U}$ depending on her uncertainty attitudes $U$ and on her initial wealth $w_{0} \in \mathbb{R}$, and as if, by the functional $\phi_{w_{0}}^{U}$, she summarized her appraisal by considering exclusively the worst scenario in $\mathcal{M}_{1, f}$.

\section{Comparative Uncertainty Aversion}

If a decision maker with preferences $U_{1}$ and with constant initial wealth $w_{0} \in \mathbb{R}$ prefers a constant monetary payoff $x \in \mathbb{R}$ to an uncertain monetary payoff $X \in \mathcal{X}$, then a more uncertainty averse decision maker with preferences $U_{2}$ and with the same constant initial wealth $w_{0} \in \mathbb{R}$ will do the same.

Definition 3. A preference functional $U_{1}: \mathcal{X} \rightarrow \mathbb{R}$ is said to be less uncertainty averse than a preference functional $U_{2}: \mathcal{X} \rightarrow \mathbb{R}$ if

$$
U_{1}\left(w_{0}+x\right) \geq U_{1}\left(w_{0}+X\right) \Rightarrow U_{2}\left(w_{0}+x\right) \geq U_{2}\left(w_{0}+X\right)
$$

for all $X \in \mathcal{X}, x \in \mathbb{R}$, and $w_{0} \in \mathbb{R}$.

\section{Theorem 2. The following statements are equivalent.}

- $U_{1}$ is less uncertainty averse than $U_{2}$.

- $\pi_{w_{0}}^{U_{1}} \leq \pi_{w_{0}}^{U_{2}}$ for all $w_{0} \in \mathbb{R}$

- $\phi_{w_{0}}^{U_{1}} \leq \phi_{w_{0}}^{U_{2}}$ for all $w_{0} \in \mathbb{R}$

Corollary 1. The following statements are equivalent.

- $U_{1}$ is less uncertainty averse than $U_{2}$.

- $R_{w_{0}}^{U_{1}} \leq R_{w_{0}}^{U_{2}}$ for all $w_{0} \in \mathbb{R}$.

- $\alpha_{w_{0}}^{U_{1}} \geq \alpha_{w_{0}}^{U_{2}}$ for all $w_{0} \in \mathbb{R}$.

Theorem 2 implies that, for instance, a more uncertainty averse decision maker would pay more money to receive insurance, and would demand more money to provide insurance, at every level of constant initial wealth $w_{0} \in \mathbb{R}$

\section{Increasing Uncertainty Aversion}

If an increasingly uncertainty averse decision maker with preferences $U$ prefers a constant monetary payoff $x \in \mathbb{R}$ to an uncertain monetary payoff $X \in \mathcal{X}$ when her constant initial wealth is $w_{1} \in \mathbb{R}$, then she will do the same when her constant initial wealth is increased to $w_{2} \in \mathbb{R}$. Definition 4. A preference functional $U: \mathcal{X} \rightarrow \mathbb{R}$ is said to be increasingly uncertainty averse if

$$
U\left(w_{1}+x\right) \geq U\left(w_{1}+X\right) \Rightarrow U\left(w_{2}+x\right) \geq U\left(w_{2}+X\right)
$$

for all $X \in \mathcal{X}, x \in \mathbb{R}$, and $w_{1}, w_{2} \in \mathbb{R}$ such that $w_{2} \geq w_{1}$

Corollary 2. The following statements are equivalent.

- $U$ is increasingly uncertainty averse.

- $\pi_{w_{1}}^{U} \leq \pi_{w_{2}}^{U}$ for all $w_{1}, w_{2} \in \mathbb{R}$ such that $w_{2} \geq w_{1}$ - $\phi_{w_{1}}^{U} \leq \phi_{w_{2}}^{U}$ for all $w_{1}, w_{2} \in \mathbb{R}$ such that $w_{2} \geq w_{1}$

Corollary 3. The following statements are equivalent.

- $U$ is increasingly uncertainty avers

- $R_{w_{1}}^{U} \leq R_{w_{2}}^{U}$ for all $w_{1}, w_{2} \in \mathbb{R}$ such that $w_{2} \geq w_{1}$

- $\alpha_{w_{1}}^{U} \geq \alpha_{w_{2}}^{U}$ for all $w_{1}, w_{2} \in \mathbb{R}$ such that $w_{2} \geq w_{1}$.

Corollary 2 implies that, for instance, an increasingly uncertainty averse decision maker would pay more money to receive insurance, and would demand more money to provide insurance, at higher levels of constant initial wealth $w_{0} \in \mathbb{R}$

\section{Cash-Subadditivity}

A decision maker's increasing uncertainty aversion determines how her choice between an uncertain monetary payoff $X \in \mathcal{X}$ and a constant monetary payoff $x \in \mathbb{R}$ is altered if a positive constant amount of money $m \in[0,+\infty)$ is added to both alternatives.

Remark 1. A preference functional $U: \mathcal{X} \rightarrow \mathbb{R}$ is increasingly uncertainty averse if and only if

$U\left(w_{0}+x\right) \geq U\left(w_{0}+X\right) \Rightarrow U\left(w_{0}+x+m\right) \geq U\left(w_{0}+X+m\right)$

for all $X \in \mathcal{X}, x \in \mathbb{R}, w_{0} \in \mathbb{R}$, and $m \in[0,+\infty)$.

Theorem 3. A preference functional $U: \mathcal{X} \rightarrow \mathbb{R}$ is increasingly uncertainty averse if and only if

$$
\pi_{w_{0}}^{U}(X+m) \geq \pi_{w_{0}}^{U}(X)-m
$$

for all $X \in \mathcal{X}, w_{0} \in \mathbb{R}$, and $m \in[0,+\infty)$

Corollary 4. A preference functional $U: \mathcal{X} \rightarrow \mathbb{R}$ is increasingly uncertainty averse if and only if

$$
R_{w_{0}}^{U}(x+m, Q) \leq R_{w_{0}}^{U}(x, Q)+m
$$

for all $(x, Q) \in \mathbb{R} \times \mathcal{M}_{1, f}$ and $m \in[0,+\infty)$.

Theorem 3 implies that if a positive constant amount of money $m \in$ $[0,+\infty)$ is added to an uncertain monetary payoff $X \in \mathcal{X}$, then the maximum price that an increasingly uncertainty averse decision maker would pay to avoid $X \in \mathcal{X}$ is decreased by less than $m \in[0,+\infty)$.

\section{Conclusion}

The indifference buver price and the indifference seller price are derived from the preferences of an uncertainty averse decision maker. The indifference buyer price is a quasiconvex risk measure, and the indifference seller price is a cash-additive convex risk measure. A decision maker is more uncertainty averse than another if and only if her indifference prices are pointwise larger than the other's. A decision maker is increasingly (respectively, decreasingly, constantly) uncertainty averse if and only if her indifference prices are increasing (respectively, decreasing, constant) functions of her constant initial wealth. Equivalently, a decision maker is increasingly (respectively, decreasingly, constantly) uncertainty averse if and only if her indifference buyer price is cash-subadditive (respectively, cash-superadditive, cash-additive).

\section{References}

S. Cerreia Vioglio, F. Maccheroni, M. Marinacci, and L. Montrucchio Uncertainty Averse Preferences. Carlo Alberto Notebook No. 77. September 2008a.

S. Cerreia Vioglio, F. Maccheroni, M. Marinacci, and L. Montrucchio. Complete Monotone Quasiconcave Duality. Carlo Alberto Notebook No. 80, November 2008b. 